\title{
Studying Ranking-Incentivized Web Dynamics
}

\author{
Ziv Vasilisky \\ zivvasilisky@campus.technion.ac.il \\ Technion
}

\author{
Moshe Tennenholtz \\ moshet@ie.technion.ac.il \\ Technion
}

\author{
Oren Kurland \\ kurland@technion.ac.il \\ Technion
}

\begin{abstract}
The ranking incentives of many authors of Web pages play an important role in the Web dynamics. That is, authors who opt to have their pages highly ranked for queries of interest often respond to rankings for these queries by manipulating their pages; the goal is to improve the pages' future rankings. Various theoretical aspects of this dynamics have recently been studied using game theory. However, empirical analysis of the dynamics is highly constrained due to lack of publicly available datasets. We present an initial such dataset that is based on TREC's ClueWeb09 dataset. Specifically, we used the WayBack Machine of the Internet Archive to build a document collection that contains past snapshots of ClueWeb documents which are highly ranked by some initial search performed for ClueWeb queries. Temporal analysis of document changes in this dataset reveals that findings recently presented for small-scale controlled ranking competitions between documents' authors also hold for Web data. Specifically, documents' authors tend to mimic the content of documents that were highly ranked in the past, and this practice can result in improved ranking.
\end{abstract}

\section{CCS CONCEPTS}

- Information systems $\rightarrow$ Information retrieval; Search engine architectures and scalability;

\section{KEYWORDS}

adversarial IR

\section{ACM Reference Format:}

Ziv Vasilisky, Moshe Tennenholtz, and Oren Kurland. 2020. Studying RankingIncentivized Web Dynamics. In Proceedings of the 43rd International ACM SIGIR Conference on Research and Development in Information Retrieval (SIGIR '20), July 25-30, 2020, Virtual Event, China. ACM, New York, NY, USA, 4 pages. https://doi.org/10.1145/3397271.3401300

\section{INTRODUCTION}

The Web is a dynamic retrieval setting. An important part of this dynamics is due to the ranking incentives of Web pages' authors. That is, some are interested in having their pages highly ranked for queries of interest. As a result, they might respond to induced rankings by modifying their documents so as to improve their future ranking - a practice also known as search engine optimization [10].

Permission to make digital or hard copies of all or part of this work for personal or classroom use is granted without fee provided that copies are not made or distributed for profit or commercial advantage and that copies bear this notice and the full citation on the first page. Copyrights for components of this work owned by others than the author(s) must be honored. Abstracting with credit is permitted. To copy otherwise, or republish, to post on servers or to redistribute to lists, requires prior specific permission and/or a fee. Request permissions from permissions@acm.org.

SIGIR '20, July 25-30, 2020, Virtual Event, China

(C) 2020 Copyright held by the owner/author(s). Publication rights licensed to ACM ACM ISBN 978-1-4503-8016-4/20/07 ..\$15.00

https://doi.org/10.1145/3397271.3401300
Recently, there has been a growing research interest in analyzing the dynamics just described using game theory $[17]^{1}$. For example, it was shown that the probability ranking principle [16] is not optimal in competitive retrieval settings as it leads to a reduced topical diversity in the corpus [2]. In addition, the document manipulation strategies of documents' authors were analyzed using game theory [15]. The robustness of induced rankings under adversarial document modifications has also been explored [9].

However, it seems that to empirically analyze the ranking incentivized dynamics of the Web, access to the query logs of commercial search engines is called for [17]. Even with such access allowed, isolating and studying specific aspects of the dynamics can be a difficult challenge [17]. As a result of this state-of-affairs, there have been recent small-scaled controlled studies of ranking competitions between incentivized documents' authors [15]. Yet, increasing the scale of such studies and performing analysis of real Web dynamics driven by induced rankings still remains a challenge [17].

To address the challenge(s) just mentioned, we have developed a novel dataset available at https://github.com/hscw09dataset/hscw09. Specifically, we used the WayBack Machine of the Internet Archive (https://archive.org/web/) to find past versions of a selected set of documents in TREC's ClueWeb09 collection; this is a Web crawl from 2009 which serves as a standard testbed for Web retrieval evaluation. The selected documents were the most highly ranked in response to ClueWeb09 queries.

Analysis of the dataset reveals that some characteristics of the temporal changes of ClueWeb09 documents are in accordance with those which have emerged from analysis of small-scaled controlled ranking competitions between students who authored short plaintext documents [15]. Thus, while we have no concrete evidence that the authors of these documents were incentivized to rank-promote them for ClueWeb09 queries, aspects of the actual modifications do conform to those applied in a competitive ranking setting by rank-incentivized documents' authors. A case in point, documents which were promoted to the highest rank for a given query became more similar to those highly ranked for the query in the past. The rationale for this competitive modification strategy in a setting with undisclosed ranking function whose induced rankings can be observed was recently provided using a game theoretic analysis [15]. This theoretical finding was supported by the analysis of controlled ranking competitions between students [15].

We believe that the dataset we have developed, and its future potential developments, will help to better understand and further explore Web dynamics with respect to the inherent competitive retrieval setting which is driven by rank-incentivized authors.

\section{RELATED WORK}

Most past work on adversarial IR has focused on spamming [5]. Search engine optimization needless necessary be black-hat or

\footnotetext{
${ }^{1}$ Similar dynamics in recommendation systems was studied in [3]
} 
spamming [10]. In fact, legitimate (a.k.a. white hat) document modifications and rank-promotion strategies play an important role in driving the dynamics of the Web retrieval setting [10]. One of our goals in developing the dataset is to study this white hat dynamics.

There is work on predicting and analyzing changes of Web pages; e.g., $[13,14]$. These studies are for general dynamics and not for ranking-oriented dynamics which we address in this paper.

There has also been work on using the past versions of a Web page so as to improve its current representation for ranking purposes $[1,8,12]$. Analysis of temporal document changes as that we present here was not reported. Still, we believe that our dataset will help to facilitate and further this line of research as well.

\section{THE DATASET}

Our first goal was to create a dataset that allows to study the temporal dynamics of documents that are highly ranked for queries. To this end, we used TREC's ClueWeb09 dataset [6]. The titles of topics 1-200 served as queries. Krovetz stemming was applied to queries and documents. We applied standard language-model-based retrieval for each query. Specifically, we used the KL divergence between the unsmoothed maximum likelihood estimate induced from the query and the Dirichlet smoothed document language models for ranking; the smoothing parameter was set to 1000 [11] We scanned each retrieved list top down and removed documents whose Waterloo's spam score [7] was below 50 until we have accumulated 50 documents. We use $L_{0}^{q}$ to denote the retrieved list for query $q$; 0 is the timestamp. The set of all documents in the lists retrieved for the 200 queries is referred to as the base set; it contains 9986 different documents. (Some documents are among the top-retrieved for more than one query.)

The next step was to collect past versions of the documents in $L_{0}^{q}$ for each query $q$. To that end, we used the WayBack Machine of the Internet Archive (https://archive.org/web/). The Internet Archive is a large repository of Web pages crawlled in different points in time. Since ClueWeb09 is a crawl from 2009, we used past versions from 2008. Specifically, we used 12 time intervals, each corresponds to a calendar month in 2008. We used a document's earliest snapshot in an interval as its version for the interval in case a snapshot existed. Thus, we consider at most 13 versions of a document: the ClueWeb version (\# 0) and the 12 past versions from 2008. The 2008 document lists for query $q$ are denoted $L_{-1}^{q}, \ldots, L_{-12}^{q}$ where -1 corresponds to the December interval and -12 corresponds to the January interval. The number of documents in each of these lists $(\leq 50)$ depends on the number of documents from $L_{0}^{q}$ with at least one past snapshot in the corresponding interval. In what follows, we use $d_{i}^{q}$ where $i \in\{0,-1, \ldots,-12\}$ to denote a document in list $L_{i}^{q}$.

Out of the 9986 different documents in the base set, 7425 (74.4\%) have at least one past version in one of the twelve 2008 time intervals. The mean number of past versions of a document is 3.06 and the median is 2 . For documents with at least one past version, the mean is 4.1 and the median is 3 .

\section{TEMPORAL DOCUMENT CHANGES}

Figure 1 presents the average similarity between documents in the base set and their past versions. Specifically, for each time interval,

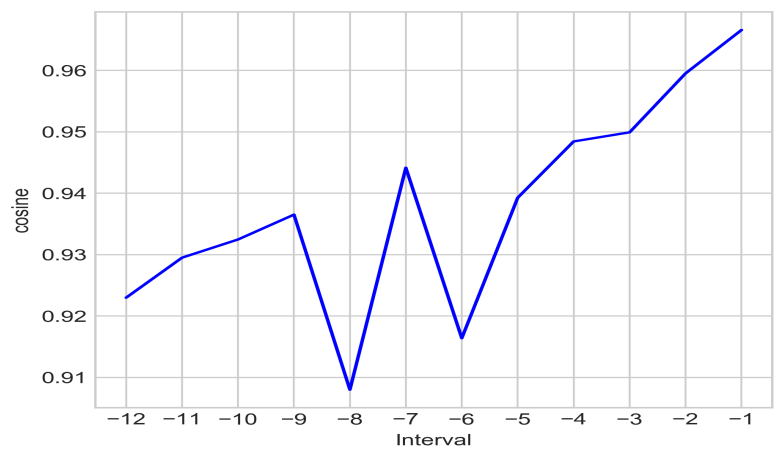

Figure 1: Average cosine similarity between a document at time interval 0 (i.e., the ClueWeb09 document) and its past version (if exists) at time interval $i$.

we compute the average cosine between the tf.idf vectors representing documents available in the interval and those representing their 0 versions (i.e., the original ClueWeb09 versions). As could be expected, we see in Figure 1 a general upward trend: the past versions of documents gradually become more similar (with a few exceptions) to their 0 versions.

We next analyze some characteristics of the changes of documents in our dataset along time. Our analysis is inspired by the ranking competitions that Raifer et al. [15] organized between students. The students wrote plaintext documents of up to 150 terms and manipulated them along time so as to have them highly ranked for queries of interest by an unknown ranking function. Raifer et al. [15] found that the documents had an increasing number of query term occurrences along time, a reduced number of stopwords, and a decreased term distribution entropy which attests to some extent to reduced content breadth [4].

We use a few definitions. The ratio of query terms that appear in a document is referred to as QueryTermsRatio. The relative query terms ratio of document $d_{i}^{q}$ with respect to its 0 version, $d_{0}^{q}$, is denoted $\operatorname{rqtr}\left(\mathbf{d}_{\mathbf{i}}^{\mathbf{q}}\right)$. The ratio of stopwords to non-stopwords in a document is denoted StopwordsRatio ${ }^{2}$. The relative stopwords ratio with respect to version 0 of document $d_{i}^{q}$ is denoted $\operatorname{rswr}\left(\mathbf{d}_{\mathbf{i}}^{\mathbf{q}}\right)$. High stopword occurrence in documents was shown to be an effective document relevance prior which corresponds to the premise that stopword presence attests to document quality [4]. The entropy of a document's term distribution, henceforth Entropy, potentially attests to its content breadth [4]. The relative entropy of document $d_{i}^{q}$, with respect to its 0 version, is denoted $\operatorname{rent}\left(\mathbf{d}_{\mathbf{i}}^{\mathbf{q}}\right)$.

Figure 2 shows that all the average (per interval) relative queryterm ratios for documents are negative; i.e., past versions of the ClueWeb09 documents have lower query terms ratio. Furthermore, we see an overall upward trend which attests to increased use of terms of queries for which the documents were highly ranked. Thus, it seems as if there is some "effort" in promoting in rankings these documents for the ClueWeb09 queries although there is clearly no

\footnotetext{
${ }^{2}$ We used the NLTK (https://www.nltk.org/) stopword list.
} 


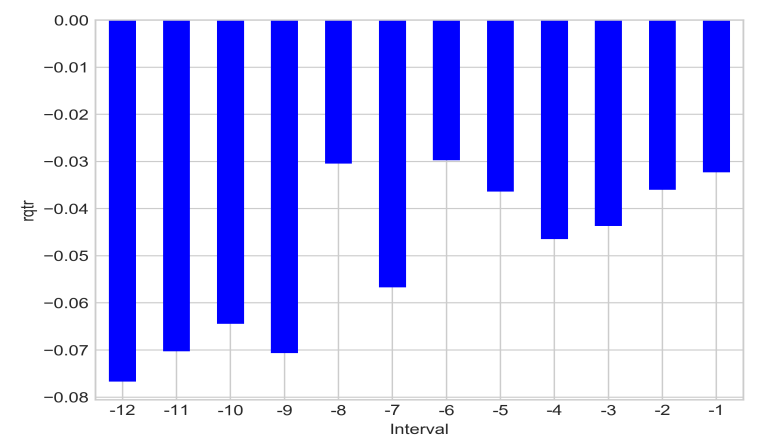

Figure 2: The average relative query terms ratio ( $r q t r)$.

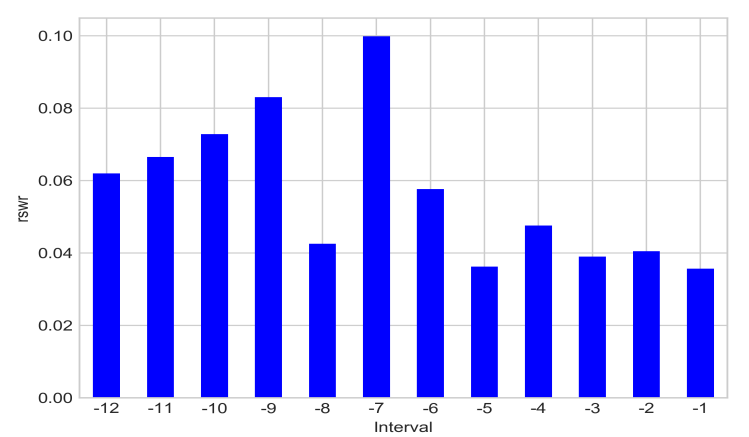

Figure 3: The average relative stopword ratio ( $r s w r)$.

explicit evidence. We note that this trend was also observed in the ranking competitions of Raifer et al. [15] where students explicitly worked on rank-promoting their documents for given queries.

We see in Figure 3 that the average, per-interval, relative stopword ratio $(r s w r)$ is positive for all intervals. That is, the stopword ratio of past versions of the ClueWeb09 documents was higher than that for the ClueWeb09 versions. We also observe in Figure 3 a general downward trend from interval -7 (June 2008) to interval -1 (December 2008). This finding echoes those from Raifer et al.'s [15] controlled ranking competitions with plaintext documents.

Figure 4 shows that for most intervals, the past versions of ClueWeb09 documents had higher entropy than that for the ClueWeb09 versions. This entropy drop (between the past and the ClueWeb09 version), which attests to decreased content breadth, is in line with Raifer et al.'s findings [15].

To summarize, although our dataset is composed of real Web documents, and we have no explicit evidence that their authors were actually trying to promote them for ClueWeb09 queries, the temporal changes of the documents are similar in several respects to those observed for short plaintext documents used in controlled ranking competitions with explicit rank-promotion incentives of their authors [15].

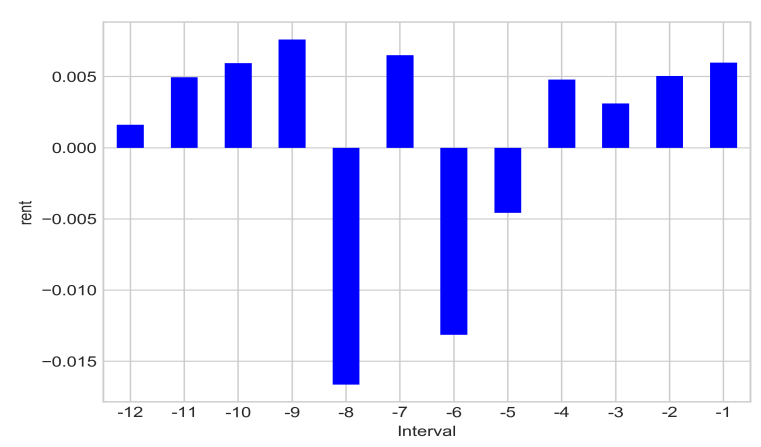

Figure 4: The average relative entropy (rent).

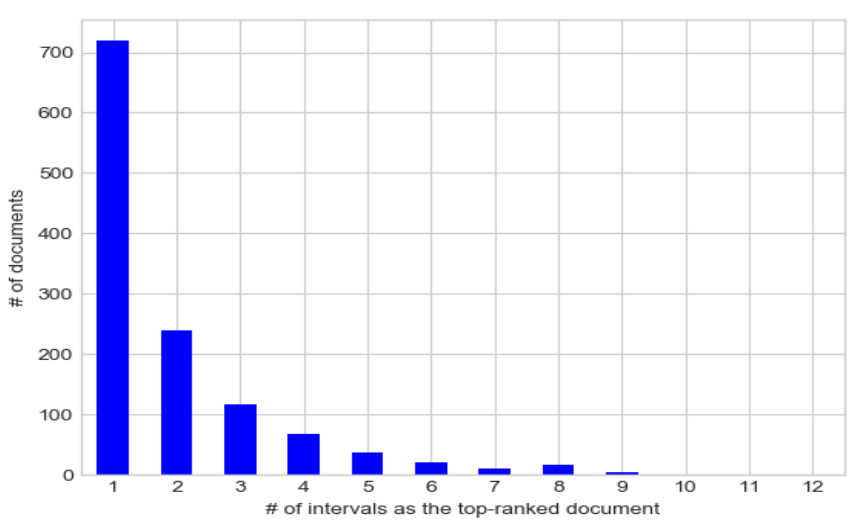

Figure 5: The number of documents in the base set whose past versions are the highest ranked for $x$ intervals.

\section{DOCUMENTS' TEMPORAL CHANGES FROM A RANKING PERSPECTIVE}

We next analyze some changes of rankings throughout the time intervals. In addition, we study the documents' changes from a ranking perspective. Recall that we use standard language-model-based retrieval. Our goal is to focus on content changes of documents rather than explore changes to anchor text, hyperlinks, etc.

In Figure 5 we present the number of documents in the ClueWeb09 base set whose past versions are ranked at the highest rank for $x$ intervals. We see that very few documents have past versions that are positioned at the highest rank for more than very few intervals. In other words, there is significant dynamics in terms of the highest rank along time. This dynamics is reminiscent of that observed by Raifer et al. [15] in their controlled ranking competitions.

Strategic manipulations?. Raifer et al. [15] analyzed - theoretically and empirically - the document manipulation strategies employed by students in their controlled competitions. As noted above, we have no explicit evidence that authors of ClueWeb09 pages were actually incentivized to rank-promote their documents for the ClueWeb09 queries. Nevertheless, the findings we presented 
above with regard to the characteristics of documents' changes attested to the fact that the manipulations of the documents along time did correspond to those observed in Raifer et al.'s competitions for rank-incentivized authors. Hence, we now further address the characterization of the manipulations from a ranking perspective using the analysis proposed by Raifer et al. [15].

We term the document ranked at the highest rank at some time interval the winner of the interval; all other documents in the interval are referred to as losers [15]. We study two groups of documents: winners $(\mathbf{W})$ of intervals and those which were losers $(\mathbf{L})$ for at least three consecutive intervals before a current interval in which they became winners. We contrast the two groups with respect to four features. The first three are those used in Section 4 : QueryTermsRatio, StopwordsRatio and Entropy. The fourth feature is the similarity to the previous winner (SimPW): the cosine similarity between the tf.idf vector representing the document and the vector representing the document which was the highest ranked in the previous interval. Following Raifer et al.'s analysis [15], we further divide the $\mathrm{L}$ group to two with respect to each feature: those whose feature value three intervals before winning was less or equal $(\mathrm{L} \leq \mathrm{W})$ or higher $(\mathrm{L}>\mathrm{W})$ than that of the winner of that interval.

We see in Figure 6 that the values of the QueryTermsRatio, StopwordsRatio and Entropy features remain relatively stable for winners. In contrast, these feature values for the two groups of losers who became winners can quite fluctuate. More importantly, we observe an upward trend of SimPW for both groups of losers. This means that losers were making their documents more similar to those of winners from the previous interval. This strategy was theoretically motivated for rank-incentivized authors by using a game theoretic modeling of the competitive retrieval setting [15]. Furthermore, the empirical finding is the same as that of Raifer et al.'s [15] for their controlled ranking competitions.

\section{CONCLUSIONS AND FUTURE WORK}

We described a dataset we have created which contains past versions of ClueWeb09 documents that are highly ranked for ClueWeb09 queries. Analysis of the dataset revealed that aspects of temporal changes of documents are in accordance with those reported for small-scale controlled ranking competitions between students who authored and manipulated short plaintext documents [15].

We plan to further develop this dataset and use it to extend our study of the Web dynamics which revolves around rankings induced for queries.

Acknowledgments. We thank the reviewers for their comments. The work by Moshe Tennenholtz was supported by funding from the European Research Council (ERC) under the European Union's Horizon 2020 research and innovation programme (grant agreement 740435).

\section{REFERENCES}

[1] Ablimit Aji, Yu Wang, Eugene Agichtein, and Evgeniy Gabrilovich. 2010. Using the past to score the present: extending term weighting models through revision history analysis. In Proc. of CIKM. 629-638.

[2] Ran Ben-Basat, Moshe Tennenholtz, and Oren Kurland. 2017. A Game Theoretic Analysis of the Adversarial Retrieval Setting. F. Artif. Intell. Res. 60 (2017), 11271164 .

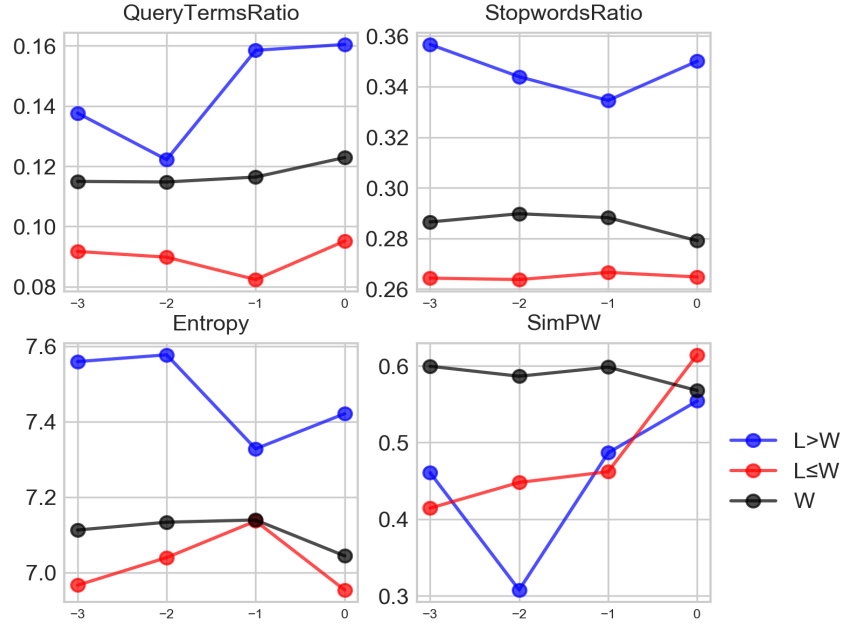

Figure 6: Average feature values per time interval: Contrasting winner documents $(\mathrm{W})$ with documents which were losers $(\mathrm{L})$ for at least three consecutive time intervals before becoming winners. $\mathrm{L}>\mathrm{W}$ and $\mathrm{L} \leq \mathrm{W}$ mark losers whose feature value was higher, or less or equal to, that of the winner three time intervals before becoming a winner.

[3] Omer Ben-Porat and Moshe Tennenholtz. 2018. A Game-Theoretic Approach to Recommendation Systems with Strategic Content Providers. In Proc. of NIPS. $1110-1120$.

[4] Michael Bendersky, W Bruce Croft, and Yanlei Diao. 2011. Quality-biased ranking of web documents. In Proc. of WSDM. 95-104.

[5] Carlos Castillo and Brian D. Davison. 2010. Adversarial Web Search. Foundations and Trends in Information Retrieval 4, 5 (2010), 377-486.

[6] Charles L. A. Clarke, Nick Craswell, and Ian Soboroff. 2009. Overview of the TREC 2009 Web Track. In Proc. of TREC.

[7] Gordon V. Cormack, Mark D. Smucker, and Charles L. A. Clarke. 2010. Efficient and Effective Spam Filtering and Re-ranking for Large Web Datasets. arXiv: 1004.5168 [cs.IR]

[8] Jonathan L. Elsas and Susan T. Dumais. 2010. Leveraging Temporal Dynamics of Document Content in Relevance Ranking. In Proc. of WSDM. 1-10.

[9] Gregory Goren, Oren Kurland, Moshe Tennenholtz, and Fiana Raiber. 2018. Ranking Robustness Under Adversarial Document Manipulations. In Proc. of SIGIR. 395-404.

[10] Zoltán Gyöngyi and Hector Garcia-Molina. 2005. Web Spam Taxonomy. In Proc. of AIRWeb 2005. 39-47.

[11] John D. Lafferty and Chengxiang Zhai. 2001. Document language models, query models, and risk minimization for information retrieval. In Proc. of SIGIR. 111119.

[12] Sérgio Nunes, Cristina Ribeiro, and Gabriel David. 2011. Term Weighting Based on Document Revision History. JASIST 62 (12 2011), 2471-2478.

[13] Kira Radinsky and Paul N. Bennett. 2013. Predicting content change on the web. In Proc. of WSDM. 415-424.

[14] Kira Radinsky, Fernando Diaz, Susan T. Dumais, Milad Shokouhi, Anlei Dong, and Yi Chang. 2013. Temporal web dynamics and its application to information retrieval. In Proc. of WSDM. 781-782.

[15] Nimrod Raifer, Fiana Raiber, Moshe Tennenholtz, and Oren Kurland. 2017. Information Retrieval Meets Game Theory: The Ranking Competition Between Documents' Authors. In Proc. of SIGIR. 465-474.

[16] Stephen E. Robertson. 1977. The Probability Ranking Principle in IR. Journal of Documentation (1977), 294-304. Reprinted in K. Sparck Jones and P. Willett (eds), Readings in Information Retrieval, pp. 281-286, 1997.

[17] Moshe Tennenholtz and Oren Kurland. 2019. Rethinking search engines and recommendation systems: a game theoretic perspective. Commun. ACM 62, 12 (2019), 66-75. 